\title{
Quantitative Microvessel Analysis with 3-D Super-Resolution Ultrasound and Velocity Mapping
}

\author{
Sevan Harput ${ }^{1}$, Matthieu Toulemonde ${ }^{2}$, Alessandro Ramalli ${ }^{3}$, Kirsten Christensen-Jeffries ${ }^{4}$, Enrico Boni ${ }^{3}$, \\ Piero Tortoli ${ }^{3}$, Chris Dunsby ${ }^{5 *}$, and Meng-Xing Tang ${ }^{2 *}$ \\ ${ }^{1}$ Division of Electrical and Electronic Engineering, London South Bank University, London, SE1 0AA, UK \\ ${ }^{2}$ ULIS Group, Department of Bioengineering, Imperial College London, London, SW7 2BP, UK \\ ${ }^{3}$ Department of Information Engineering, University of Florence, 50139 Florence, IT \\ ${ }^{4}$ Biomedical Engineering Department, Division of Imaging Sciences, King's College London, SE1 7EH, London, UK \\ ${ }^{5}$ Department of Physics and the Centre for Pathology, Imperial College London, London, SW7 2AZ, UK \\ ${ }^{*}$ These authors contributed equally to this work \\ E-mail: harputs@1sbu.ac.uk, mengxing.tang@imperial.ac.uk
}

\begin{abstract}
Medical image analysis is becoming increasingly accessible in the clinic. Computed tomography (CT) or magnetic resonance imaging (MRI) scans are usually post-processed to generate a 3-D visualization of the human body for surgical assistance or extract quantitative data to provide additional diagnostic information. Three dimensional super-resolution ultrasound (SR US) imaging can provide similar information at a micro-level without the high cost or ionising radiation.

In this study, we implemented a high volumetric-rate 3-D SR US imaging with a 2-D spiral-shaped array and imaged an in vitro microvascular structure. From the 3-D SR US images clinically relevant parameters, such as microvascular flow rate, microvessel density and tortuosity, were extracted and compared with the ground truth.
\end{abstract}

\section{INTRODUCTION}

Super-resolution ultrasound (SR US) imaging with contrast agents is becoming an accepted method to improve image resolution beyond the diffraction limit. Many studies demonstrated the use of 2-D SR US imaging using microbubbles [1][15] and nanodroplets [16]-[19]. These studies only achieved 2-D SR US slices of a 3-D structure, which makes the volumetric observations more challenging or impossible since super-resolution is not present in the elevational direction. The 3-D SR US imaging can overcome the diffraction limited resolution for the whole imaging volume [20]-[28].

Medical image processing and analysis is employed in many clinical scenarios, including early diagnosis and treatment monitoring. The basic idea of this work is to further process 3D SR US images to extract important quantitative information. For this purpose, we exploited our previously developed 3-D SR US system for high volumetric imaging rate, based on a 2-D spiral-shaped array [28], [29]. This system was used to image an in vitro microvascular structure. From the 3D SR US images, individual microbubbles were tracked to identify and separate microvessels in which they were flowing through. After separating the microvessels, clinically relevant parameters were extracted from the 3-D SR US images. Microvascular flow rate, microvessel density and tortuosity were calculated for each microvessel and compared with the ground truth.

\section{MAterials AND Methods}

\section{A. Experimental Setup}

A 512-element 2-D density-tapered spiral array with a center frequency of $3.7 \mathrm{MHz}$ and a bandwidth of $60 \%$ (Vermon S.A., Tours, France) was used for 3-D imaging with the details given in [28]-[30]. Two ULA-OP 256 systems were synchronized to achieve 3-D SR US imaging by using the 2D spiral array [31], [32]. A microvascular flow phantom was fabricated with two $200 \mu \mathrm{m}$ twisted cellulose tubes in a double helix shape as shown in Fig. 1. Both tubes had constant microbubble flow velocity of $44 \mathrm{~mm} / \mathrm{s}$, but into opposite directions, driven by a dual infusion pump. A 1:2,000 (Native microbubble solution:Water) diluted Sonovue solution was flowed through the microvessel phantom during imaging. 12,000 volumetric images were acquired with a frame rate of

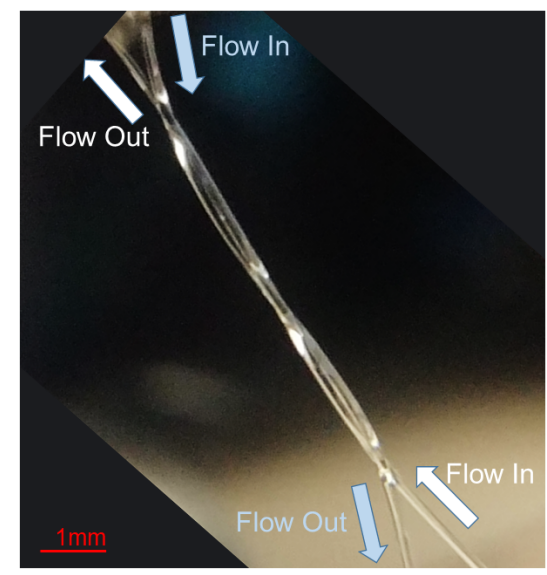

Fig. 1. Optical image of the experimental setup with two $200 \mu \mathrm{m}$ cellulose tubes arranged in a double helix pattern. 


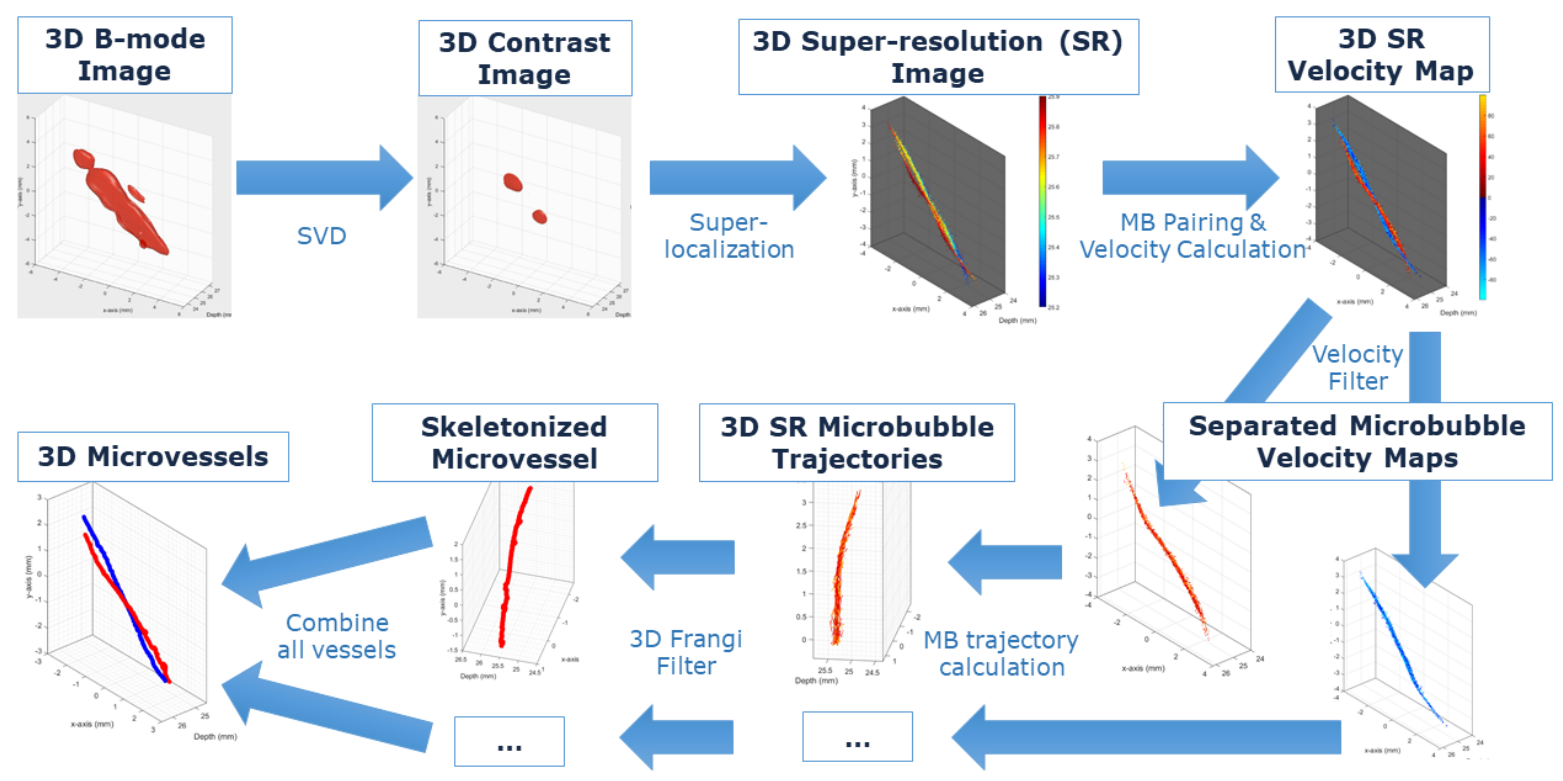

Fig. 2. Illustration of the data processing steps.

$500 \mathrm{~Hz}$ (9 compounded volumes at $4500 \mathrm{~Hz}$ ) using a MI of $0.055 . \mathrm{mm}$. The imaging wavelength was $405 \mu \mathrm{m}$.

\section{B. 3-D Data Processing}

The steps of the data processing chain are illustrated in Fig. 2.The 3-D B-mode ultrasound images were first processed with a spatio-temporal filter, based on singular value decomposition (SVD), to remove the echoes from the cellulose tubes [33], [34]. Upper and lower intensity thresholds were set to reject signals, which might be due to multiple microbubbles and noise, respectively. The super-localization of microbubbles was performed as explained in [35]. Super-localized microbubbles were tracked and paired between consecutive frames to calculate their velocities, which was done by multiplying their Euclidean distance with the frame-rate. Microvascular flow rate was estimated by the average microbubble velocity and compared with the velocity value set on the infusion pump to calculate the error. Each potential microvessel was separated with a velocity filter (a band-pass filter) based on the assumption of different flow rates and direction. A 3D Frangi filter was used to embody the microvessels for positive and negative velocity values separately [36]. After this, the filtered microvessels were skeletonized to calculate microvessel density and tortuosity. The tortuosity was calculated by dividing the length of the microvessel by the Euclidian distance between the ends of the microvessel, where both values were measured from the 3-D skeletonized images.

\section{RESULTS \& DISCUSSION}

Fig. 3 shows a single 3-D B-mode image of the microvascular phantom, which cannot be used to differentiate two cellulose tubes due to its low resolution (full-width-half-maximum of $495 \mu \mathrm{m}$ ) compared to the microvessel size (diameter of 200 $\mu \mathrm{m})$. After super-resolution processing, a total of 9,469 microbubbles were localized and 4,742 microbubble-pairs were tracked from consecutive frames. All tracked microbubbles were superimposed in a single frame to generate the 3-D superresolved velocity map shown in Fig. 4 A, where both touching tubes can be resolved.

After generating the 3-D SR velocity maps, microbubbles were separated into different groups by using the direction and velocity information, as illustrated in Fig. 2. After the velocity filter, microvascular flow was estimated in both tubes with an average flow velocity of $49.1 \mathrm{~mm} / \mathrm{s}$ (11.6\% error) and $50.7 \mathrm{~mm} / \mathrm{s}$ (15.2\% error). Again, after this velocity filter stage, microbubble velocity traces were calculated and drawn

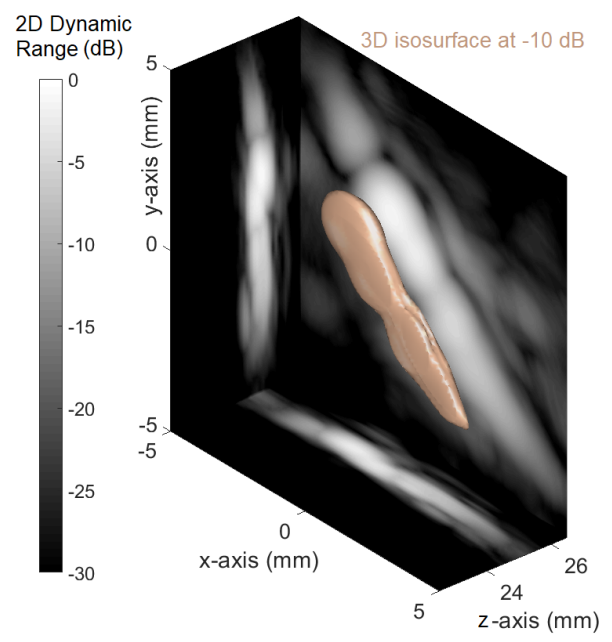

Fig. 3. 3-D ultrasound B-mode image with 2-D maximum intensity projections in $\mathrm{x}, \mathrm{y}$ and $\mathrm{z}$ directions. 

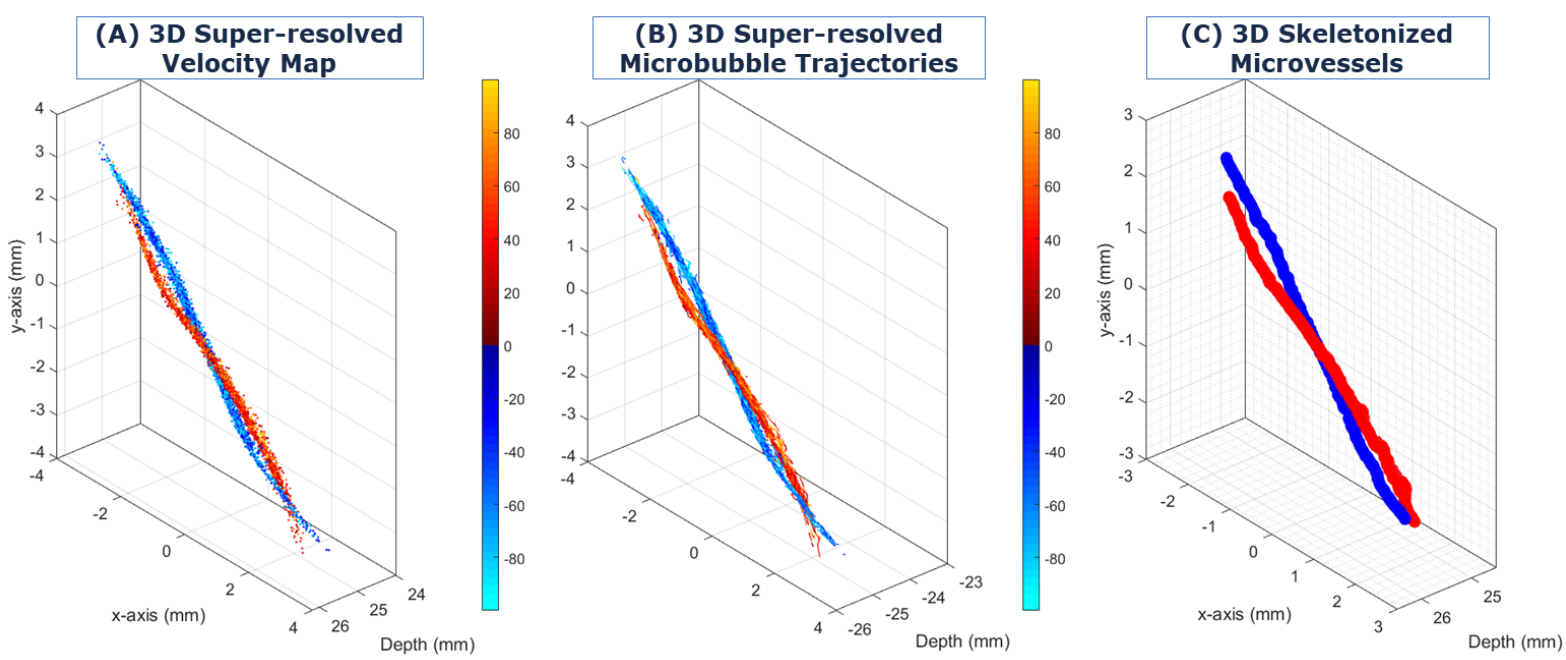

Fig. 4. (A) 3-D super-resolved velocity maps. (B) 3-D super-resolved microbubble trajectories. (C) 3-D microvessels embodied by skeletonizing microbubble trajectories.

between paired microbubbles. Although this processing was performed separately for the microbubbles flowing in different directions, all trajectories are plotted together for easy comparison in Fig. 4 B.

The microbubble trajectories were filtered with a 3-D Frangi filter to skeletonize the microvessels, which allowed the extraction of quantitative information, such as the microvessel density and tortuosity. The microvessel density was calculated as $0.3 \%$ of the SR US imaging volume $\left(8 \times 8 \times 3 \mathrm{~mm}^{3}\right)$ for the given experimental setup.

Tortuosity values were calculated from the skeletonized microvessels as 1.081 (the length of the microvessel $=6.91$ $\mathrm{mm}$ and the distance between its ends $=6.39 \mathrm{~mm}$ ) and 1.052 (the length of the microvessel $=6.24 \mathrm{~mm}$ and the distance between its ends $=5.93 \mathrm{~mm}$ ). From the optical image, the tortuosity is measured as 1.03 , but the tortuosity is expected to be underestimated from the 2-D optical projection of a 3-D microvessel. Also, the 3-D SR US images only captured the twisted part of the cellulose tubes inside the imaging region of the ultrasound probe, which can result in a higher tortuosity value.

\section{CONCLUSION}

Two $200 \mu \mathrm{m}$ tubes (smaller than half the imaging wavelength) arranged in a double helix shape were super resolved and flow velocities within these tubes were calculated. These 3-D SR US images and velocity maps were further processed to perform quantitative microvessel analysis. The 3-D Frangi filter works well around the center of the image. However, at the edges of the image, where the number of localized microbubbles is lower, the end sections of the microvessels are not skeletonized. In this non-complex microvascular phantom, tortuosity, flow velocity, flow direction and microvascular density were estimated successfully.
This work showed that 3-D SR US images are suitable for microvessel analysis and can be used to provide clinically important information by further image processing.

\section{ACKNOWLEDGMENTS}

This work was supported mainly by the EPSRC under Grant EP/N015487/1 and EP/N014855/1, in part by the Wellcome Trust (iFIND, IEH Award 102431), in part by the King's College London (KCL) and Imperial College London EPSRC Centre for Doctoral Training in Medical Imaging (EP/L015226/1), in part by the Wellcome EPSRC Centre for Medical Engineering at KCL (WT 203148/Z/16/Z), in part by the Department of Health through the National Institute for Health Research comprehensive Biomedical Research Center Award to Guy's and St Thomas' NHS Foundation Trust in partnership with KCL and King's College Hospital NHS Foundation Trust, in part by the Graham-Dixon Foundation and in part by NVIDIA GPU grant.

\section{REFERENCES}

[1] O. M. Viessmann, R. J. Eckersley, K. Christensen-Jeffries, M.-X. Tang, and C. Dunsby, "Acoustic super-resolution with ultrasound and microbubbles," Phys. Med. Biol, vol. 58, pp. 6447-6458, 2013.

[2] Y. Desailly, J. Pierre, O. Couture, and M. Tanter, "Resolution limits of ultrafast ultrasound localization microscopy," Phys. Med. Biol, vol. 60, pp. 8723-8740, 2015

[3] K. Christensen-Jeffries, R. J. Browning, M.-X. Tang, C. Dunsby, and R. J. Eckersley, "In vivo acoustic super-resolution and super-resolved velocity mapping using microbubbles," IEEE Trans. Med. Imag., vol. 34, no. 2, pp. 433-440, 2015.

[4] D. Ackermann and G. Schmitz, "Detection and tracking of multiple microbubbles in ultrasound b-mode images," IEEE Trans. Ultrason. Ferroelectr., Freq. Control, vol. 63, no. 1, pp. 72-82, 2016.

[5] A. Bar-Zion, C. Tremblay-Darveau, O. Solomon, D. Adam, and Y. C. Eldar, "Fast vascular ultrasound imaging with enhanced spatial resolution and background rejection," IEEE Trans. Med. Imag., vol. 36, pp. 169-180, 2017.

[6] J. Foiret, H. Zhang, T. Ilovitsh, L. Mahakian, S. Tam, and K. W. Ferrara, "Ultrasound localization microscopy to image and assess microvasculature in a rat kidney," Scientific Reports, vol. 7, no. 13662, pp. 1-12, 2017. 
[7] S. Harput, K. Christensen-Jeffries, Y. Li, J. Brown, R. J. Eckersley, C. Dunsby, and M.-X. Tang, "Two stage sub-wavelength motion correction in human microvasculature for ceus imaging," in IEEE International Ultrasonics Symposium (IUS), 2017, pp. 1-4.

[8] S. Harput, K. Christensen-Jeffries, J. Brown, R. J. Eckersley, C. Dunsby, and M.-X. Tang, "Localisation of multiple non-isolated microbubbles with frequency decomposition in super-resolution imaging," in IEEE International Ultrasonics Symposium (IUS), 2017, pp. 1-4.

[9] O. Couture, V. Hingot, B. Heiles, P. Muleki-Seya, and M. Tanter, "Ultrasound localization microscopy and super-resolution: A state of the art," IEEE Trans. Ultrason., Ferroelectr., Freq. Control, vol. 65, no. 8, pp. 1304-1320, 2018.

[10] P. Song, J. D. Trzasko, A. Manduca, R. Huang, R. Kadirvel, D. F. Kallmes, and S. Chen, "Improved super-resolution ultrasound microvessel imaging with spatiotemporal nonlocal means filtering and bipartite graph-based microbubble tracking," IEEE Trans. Ultrason., Ferroelectr., Freq. Control, vol. 65, no. 2, pp. 149-167, 2018.

[11] T. Opacic, S. Dencks, B. Theek, M. Piepenbrock, D. Ackermann, A. Rix, T. Lammers, E. Stickeler, S. Delorme, G. Schmitz, and F. Kiessling, "Motion model ultrasound localization microscopy for preclinical and clinical multiparametric tumor characterization," Nature Communications, vol. 9, no. 1527 , pp. 1-13, 2018.

[12] T. Ilovitsh, A. Ilovitsh, J. Foiret, B. Z. Fite, and K. W. Ferrara, "Acoustical structured illumination for super-resolution ultrasound imaging," Communications Biology, vol. 1, no. 3, 2018.

[13] S. Harput, K. Christensen-Jeffries, J. Brown, Y. Li, K. J. Williams, A. H. Davies, R. J. Eckersley, C. Dunsby, and M. Tang, "Two-stage motion correction for super-resolution ultrasound imaging in human lower limb," IEEE Trans. Ultrason., Ferroelectr., Freq. Control, vol. 66, no. 4, pp. 676-691, 2018.

[14] S. Dencks, M. Piepenbrock, T. Opacic, B. Krauspe, E. Stickeler, F. Kiessling, and G. Schmitz, "Clinical pilot application of superresolution us imaging in breast cancer," IEEE Trans. Ultrason., Ferroelectr., Freq. Control, vol. 66, no. 3, pp. 517-526, 2019.

[15] S. Harput, G. Zhang, M. Toulemonde, J. Zhu, K. Christensen-Jeffries, J. Brown, R. J. Eckersley, C. Dunsby, and M.-X. Tang, "Super-resolution ultrasound image filtering with machine-learning to reduce the localization error," in IEEE International Ultrasonics Symposium (IUS), 2019, pp. $1-4$.

[16] G. P. Luke, A. S. Hannah, and S. Y. Emelianov, "Super-resolution ultrasound imaging in vivo with transient laser-activated nanodroplets," Nano Letters, vol. 16, pp. 2556-2559, 2016.

[17] H. Yoon, K. A. Hallam, C. Yoon, and S. Y. Emelianov, "Super-resolution imaging with ultrafast ultrasound imaging of optically triggered perfluorohexane nanodroplets," IEEE Trans. Ultrason., Ferroelectr., Freq. Control, vol. 65, no. 12, pp. 2277-2285, 2018.

[18] G. Zhang, S. Harput, S. Lin, K. Christensen-Jeffries, C. H. Leow, J. Brown, C. Dunsby, R. J. Eckersley, and M.-X. Tang, "Acoustic wave sparsely activated localization microscopy (awsalm): Super-resolution ultrasound imaging using acoustic activation and deactivation of nanodroplets," Applied Physics Letters, vol. 113, no. 1, p. 014101, 2018.

[19] G. Zhang, S. Harput, H. Hu, K. Christensen-Jeffries, J. Zhu, J. Brown, C. H. Leow, R. J. Eckersley, C. Dunsby, and M.-X. Tang, "Fast acoustic wave sparsely activated localization microscopy (fast-awsalm): Ultrasound super-resolution using plane-wave activation of nanodroplets," IEEE Trans. Ultrason., Ferroelectr., Freq. Control, vol. 66, no. 6, pp. 1039-1046, 2019.

[20] M. A. O'Reilly and K. Hynynen, "A super-resolution ultrasound method for brain vascular mapping," Medical Physics, vol. 40, no. 110701, 2013.

[21] Y. Desailly, O. Couture, M. Fink, and M. Tanter, "Sono-activated ultrasound localization microscopy," Applied Physics Letters, vol. 103 no. $174107,2013$.

[22] C. Errico, J. Pierre, S. Pezet, Y. Desailly, Z. Lenkei, O. Couture, and M. Tanter, "Ultrafast ultrasound localization microscopy for deep superresolution vascular imaging," Nature, vol. 527, pp. 499-507, 2015.

[23] F. Lin, S. E. Shelton, D. Espindola, J. D. Rojas, G. Pinton, and P. A. Dayton, "3-d ultrasound localization microscopy for identifying microvascular morphology features of tumor angiogenesis at a resolution beyond the diffraction limit of conventional ultrasound," Theranostics, vol. 7, no. 1, pp. 196-204, 2017.

[24] K. Christensen-Jeffries, J. Brown, P. Aljabar, M.-X. Tang, C. Dunsby, and R. J. Eckersley, "3-d in vitro acoustic super-resolution and superresolved velocity mapping using microbubbles," IEEE Trans. Ultrason., Ferroelectr, Freq. Control, vol. 64, no. 10, pp. 1478-1486, 2017.
[25] K. Christensen-Jeffries, S. Harput, J. Brown, G. Zhang, J. Zhu, C. Dunsby, M.-X. Tang, and R. J. Eckersley, "3d in vitro ultrasound super-resolution imaging using a clinical system," in IEEE International Ultrasonics Symposium (IUS), 2018, pp. 1-4.

[26] J. Zhu, E. M. Rowland, S. Harput, K. Riemer, C. H. Leow, B. Clark, K. Cox, A. Lim, K. Christensen-Jeffries, G. Zhang, J. Brown, C. Dunsby, R. J. Eckersley, P. D. Weinberg, and M.-X. Tang, "3d super-resolution us imaging of rabbit lymph node vasculature in vivo by using microbubbles," Radiology, vol. 291, no. 3, pp. 642-650, 2019, pMID: 30990382.

[27] B. Heiles, M. Correia, V. Hingot, M. Pernot, J. Provost, M. Tanter, and O. Couture, "Ultrafast 3d ultrasound localization microscopy using a $32 \times 32$ matrix array," IEEE Trans. Med. Imag., vol. 38, no. 9, pp. 2005$2015,2019$.

[28] S. Harput, K. Christensen-Jeffries, A. Ramalli, J. Brown, J. Zhu, G. Zhang, C. H. Leow, M. Toulemonde, E. Boni, P. Tortoli, R. J. Eckersley, C. Dunsby, and M.-X. Tang, "3-d super-resolution ultrasound imaging with a 2-d sparse array," IEEE Trans. Ultrason., Ferroelectr. Freq. Control, vol. 67, no. 2, pp. 269-277, 2020.

[29] S. Harput, K. Christensen-Jeffries, J. Brown, J. Zhu, G. Zhang, C. H. Leow, M. Toulemonde, A. Ramalli, E. Boni, P. Tortoli, R. J. Eckersley, C. Dunsby, and M.-X. Tang, "3-d super-resolution ultrasound imaging using a 2-d sparse array with high volumetric imaging rate," in IEEE International Ultrasonics Symposium (IUS), 2018, pp. 1-4.

[30] A. Ramalli, E. Boni, A. S. Savoia, and P. Tortoli, "Density-tapered spiral arrays for ultrasound 3-d imaging," IEEE Trans. Ultrason., Ferroelectr. Freq. Control, vol. 62, no. 8, pp. 1580-1588, 2015.

[31] E. Boni, L. Bassi, A. Dallai, F. Guidi, V. Meacci, A. Ramalli, S. Ricci, and P. Tortoli, "Ula-op 256: A 256-channel open scanner for development and real-time implementation of new ultrasound methods," IEEE Trans. Ultrason., Ferroelectr., Freq. Control, vol. 63, no. 10, pp. 14881495, 2016.

[32] E. Boni, L. Bassi, A. Dallai, V. Meacci, A. Ramalli, M. Scaringella, F. Guidi, S. Ricci, and P. Tortoli, "Architecture of an ultrasound system for continuous real-time high frame rate imaging," IEEE Trans. Ultrason., Ferroelectr, Freq. Control, vol. 64, no. 9, pp. 1276-1284, 2017.

[33] C. Demene, T. Deffieux, M. Pernot, B.-F. Osmanski, V. Biran, J.-L. Gennisson, L.-A. Sieu, A. Bergel, S. Franqui, J.-M. Correas, I. Cohen, O. Baud, and M. Tanter, "Spatiotemporal clutter filtering of ultrafast ultrasound data highly increases doppler and fultrasound sensitivity," IEEE Trans Med Imaging, vol. 34, no. 11, pp. 2271-2285, 2015.

[34] J. Brown, K. Christensen-Jeffries, S. Harput, G. Zhang, J. Zhu, C. Dunsby, M. Tang, and R. J. Eckersley, "Investigation of microbubble detection methods for super-resolution imaging of microvasculature," IEEE Trans. Ultrason., Ferroelectr., Freq. Control, vol. 65, no. 5, pp. 803-814, 2019.

[35] K. Christensen-Jeffries, S. Harput, J. Brown, P. N. T. Wells, P. Aljabar, C. Dunsby, M.-X. Tang, and R. J. Eckersley, "Microbubble axial localization errors in ultrasonic super-resolution imaging," IEEE Trans. Ultrason., Ferroelectr., Freq. Control, vol. 64, no. 11, pp. 1644-1654, 2017.

[36] A. Frangi, W. Niessen, K. Vincken, and M. Viergever, "Multiscale vessel enhancement filtering," in MICCAI, 1998, pp. 130-137. 